\title{
Using Ultracapacitors in Photovoltaic Systems. A technical proposal
}

\author{
José Antonio Domínguez Vázquez, Fernando Martínez Rodrigo, José Miguel Ruiz and Santiago Lorenzo \\ Matilla \\ Department of Electronic Technology \\ E.T.S.I.I. Valladolid University \\ Paseo del Cauce s/n, 47011 Valladolid, Spain. \\ Phone +34 9834233 38, fax +34 9834233 10, e-mail: josdom@eis.uva.es
}

\section{Introduction}

In this paper we expose a new strategy to deliver the power of a Photovoltaic (or any other energy source of small power) System attached to the main. The object of the implementation of a battery of ultracapacitors in the photovoltaic system is to convert a variable power delivery to constant power. This will improve the energy efficiency by two ways. One is to take advantage of the time in which the system does not work because the power flow is too low (irradiation). Another advantage is that the output power is lower than input power. The last affirmation is obvious showing the next figure 1 :

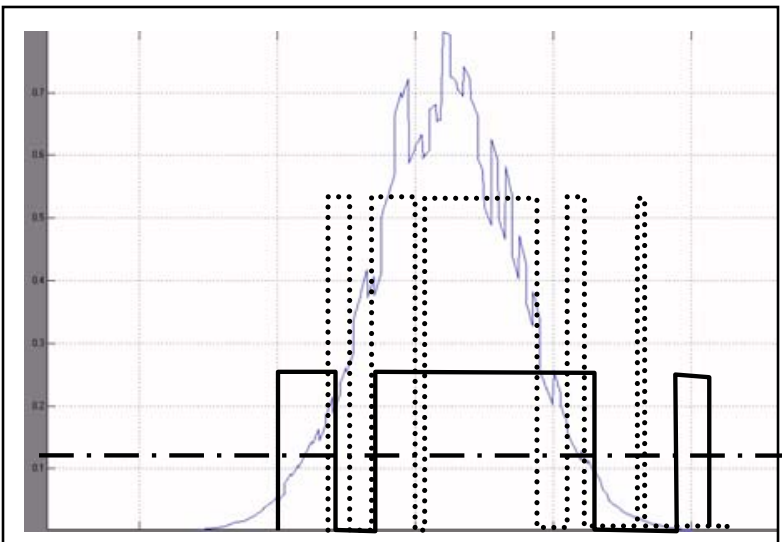

Figure 1: Several strategies depending of output power inverter.

1. Input power of the renewable power plant (photovoltaic Panels in this case)

2. .... Output power of inverter P1

3. _ Output power of inverter P2 such $P 2<P 1$

4. --- Output power of inverter $P 3$ such $P 3<P 2<P 1$

It's obvious that input energy integer value can be obtained by several ways. So we can study which output power is the best choice for our energy source choosing an the inverter that works the most time in its best efficiency ratio.

Key words: ultracapacitors, constant output power, equalization

\section{Modelling the system.}

\section{A. Irradiance modelling}

We need to know the basic equations of our energy source. In this case, the sun irradiation. In order to simplify as possible, we can consider irradiation is modelled by a gaussian expression. Next equation 1 will show the behaviour of daily irradiation, where its maximum is 1 , and the base is 12 hours wide.

$$
G d=1 \cdot e^{\frac{-1}{2}\left[\frac{(x-12)}{\sigma_{d}}\right]^{2}}
$$

Equation 1: daily irradiation. $\sigma_{d}=2.65$

The annual irradiation is considered as a gaussian modulation. This function reaches its maximum at the half of the year and its supossed about $1000 \mathrm{~W} / \mathrm{m}^{2}$. In order to determine the variance we consider the maximum irradiance of one day. This day must be at the beginning or at the finish of the year. SO annual expression for irradiation is:

$$
G d=A \cdot e^{\frac{-1}{2}\left[\frac{(x-4380)}{\sigma_{a}}\right]^{2}}
$$

Equation 2: annual irradiation.

$$
\sigma_{d}=3000 \text { and } A=1.1 \mathrm{~kW} / \mathrm{m}^{2}
$$

Two aleatory variables will simulate the effect of the climatology conditions like clouds. The product of these four elements will provides us the annual irradiance that must verify one condition: its integer must be the measured annual irradiance.

$$
G_{\text {Total }}=G a \times G d
$$

Equation 3: Total annual irradiance.

At this moment we have to adjust some of the values calculated before. Troubles appear considering daily irradiation as a Gaussian function, where the maximum value is reached at one instant only. Actually the maximum values, specially in summer, are reached during several hours. In order to resolve this problem 
we can suppose that there are more sunny hours or that maximum values of irradiance are higher.

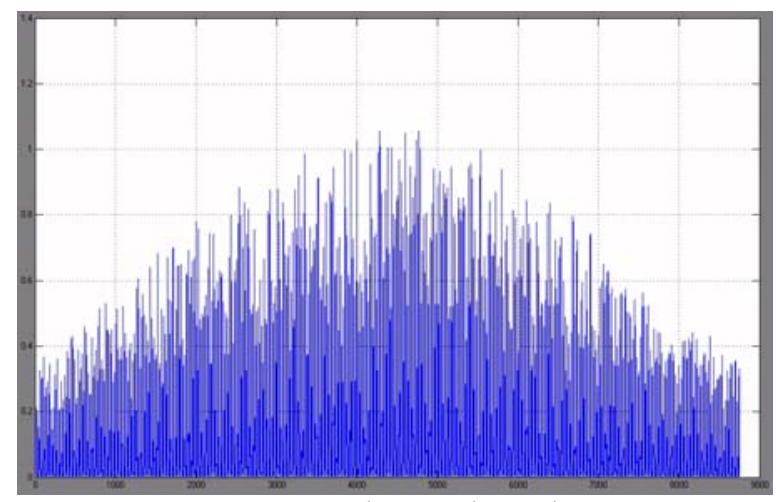

Figure 2: Total annual irradiance

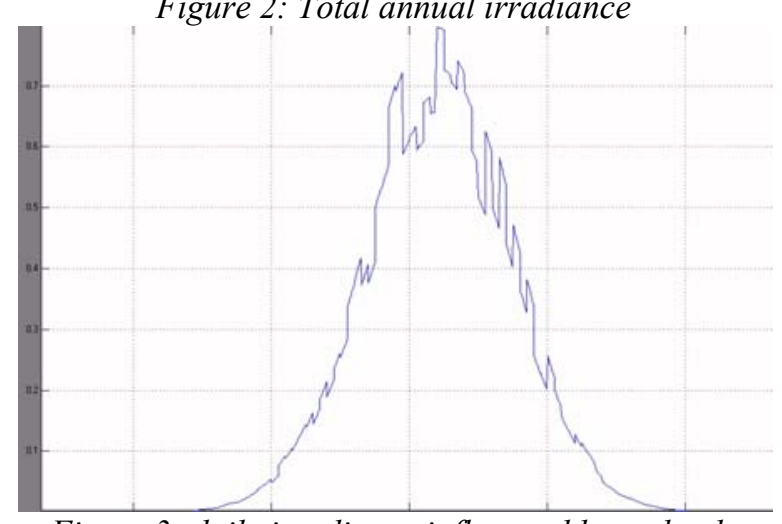

Figure 3: daily irradiance influenced by a cloudy day.

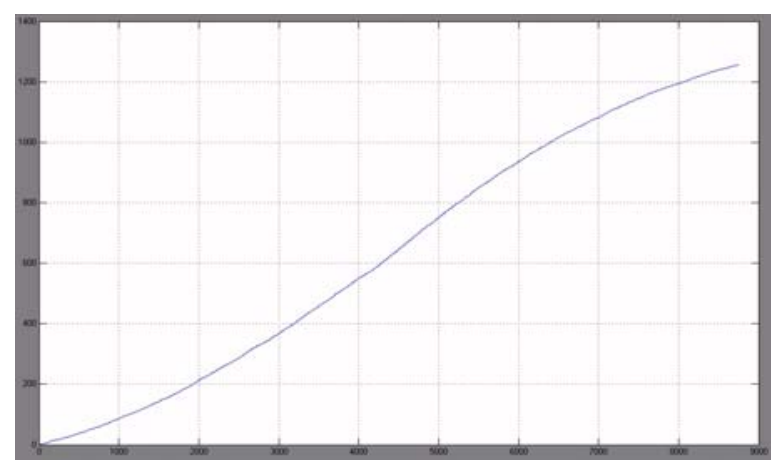

Figure 4: Total annual energy.

In spite of this small considerations, we are going to make a comparison, so the influence is going to affect at every system in the same way.

Now we are going to express every gaussian function as Fourier cosine series in order to obtain an expression of their integers helping to solve the system equations.

General expression is shown in the equation 4:

$$
f(x)=a_{0}+\sum^{n} a_{n} \cos \frac{n \pi x}{L}
$$

Equation 4: General Fourier cosine series. where:

$a_{0}=\frac{1}{L} \int_{0}^{L} f(x) d x$

$a_{n}=\frac{2}{L} \int_{0}^{L} \cos \left(\frac{n \pi x}{L}\right) f(x) d x$

We can obtain the next parameters.

For Ga:

$$
\begin{aligned}
& a_{0}=0.7346 \\
& a_{2}=-0.2926 \\
& a_{4}=-0.0382 \\
& a_{6}=-0.0168 \\
& a_{i=\text { odd }}=0
\end{aligned}
$$

For Gd

$$
\begin{aligned}
& a_{0}=0.2768 \\
& a_{2}=-0.4351 \\
& a_{4}=0.2114 \\
& a_{6}=-0.0635 \\
& a_{i=\text { odd }}=0
\end{aligned}
$$

So total irradiation is (equation 5):

$$
\begin{aligned}
G_{T} & =a_{0}^{0} a_{0}^{1}+a_{0}^{0} a_{2}^{1} \cos \frac{2 \pi x}{L_{1}}+a_{0}^{0} a_{4}^{1} \cos \frac{4 \pi x}{L_{1}}+a_{0}^{0} a_{6}^{1} \cos \frac{6 \pi x}{L_{1}}+ \\
& +a_{2}^{0} a_{0}^{1} \cos \frac{2 \pi x}{L_{0}}+a_{2}^{0} a_{2}^{1} \cos \frac{2 \pi x}{L_{0}} \cos \frac{2 \pi x}{L_{1}}+a_{2}^{0} a_{4}^{1} \cos \frac{2 \pi x}{L_{0}} \cos \frac{4 \pi x}{L_{1}}+ \\
& +a_{2}^{0} a_{6}^{1} \cos \frac{2 \pi x}{L_{0}} \cos \frac{6 \pi x}{L_{1}}+\ldots
\end{aligned}
$$

Equation 5: Total irradiation as a Fourier series

\section{B. Photovoltaic System modelling}

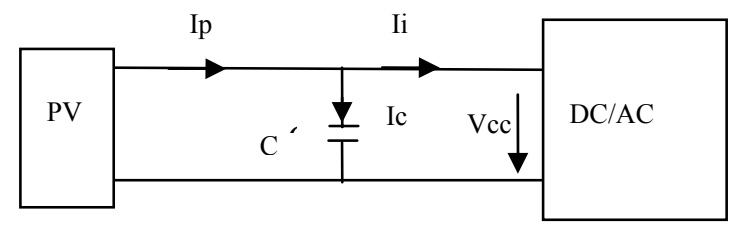

Figure 5: total system and its variables.

We consider de system showed in the figure 5, where:

- PV represents the Photovoltaic Modules

- $\quad \mathrm{C}$ is the ultracapacitors group.

- Block DC/AC is the inverter.

- Ip is the current from the PV modules, Ii is the input inverter current and Ic is the current that flow in or out of the ultrcapacitors group.

$\mathrm{Vcc}$ is the DC voltage.

We have considered internal resistance of the ultracapacitors as a very slow value, so discharge currents are unappreciated. Other losses like resistance wires are unappreciated.[2],[3] Our system will work between maximum and minimum voltages. We can consider two strategies:

- Searching the Maximum Power Point (MPP) every time. We need a DC regulator between PV modules and ultracapacitors 
- At variable voltage but no MPP searching. We work around the $10 \%$ of the MPP voltage at standard conditions.

We can calculate how many ultracapacitors have to be connected in order to reach the DC voltage:

$$
n^{\mathbf{o}} \geq \frac{V_{c c \max }}{V_{\text {cond } \max }}
$$

Then the maximum dc voltage is limited to:

$$
n^{\mathrm{o}} \times V_{\text {cond } \max }
$$

The number of parallel series is limited by the assumed cost and in a less important level, by the energy and the discharge time at constant power. Let's calculate the currents of the system. Firstly Ip, current from the PV modules, equation 6.

$$
\begin{aligned}
& I_{p}=N \cdot I_{S C P} \cdot n_{p}\left(1-e^{\frac{-e\left(V_{o c}-V_{c c}\right)}{m k T}}\right) \\
& N=\text { PVpanels } \\
& T=\text { Temperature } \\
& n_{p}=n^{\circ} \text { paralell_cells } \\
& V_{o c}=\text { Ouput_circuit_voltage } \\
& I_{\text {scp }}=\text { Short_Circuit_Current }=C_{1} \cdot G_{T} \\
& m=\text { parameter } \\
& e=\text { electron_charge } \\
& k=\text { cte.Boltzman } \\
& \quad \text { Equation 6: Current from PV modules }
\end{aligned}
$$$$
\text { Clearly: }
$$

$$
I_{p}=I_{c}+I_{i}
$$

It's time to consider that system has two states:

State 1: Ultracapacitors in charging mode: $I_{i=0}$

So: $I_{p}=I_{c}$

and:

$$
\begin{aligned}
& V_{c c}=V_{c c \min }+\frac{\# \text { series }}{C \cdot \# \text { parallel }} \int I_{p} d t= \\
& V_{c c \min }+\frac{\# \text { series }}{C \cdot \# \text { parallel }} \int N \cdot I_{S C P} \cdot n_{p}\left(1-e^{\frac{-e\left(V_{o c}-V_{c c}\right)}{m k T}}\right) d t= \\
& V_{c c \min }+\frac{\# \text { series }}{C \cdot \# \text { parallel }} \int N \cdot C_{1} \cdot G_{T} \cdot n_{p}\left(1-e^{\frac{-e\left(V_{o c}-V_{c c}\right)}{m k T}}\right) d t
\end{aligned}
$$

Equation 7: DC voltage in state 1, charging mode. We can express this equation 7 in the next way:

where

$$
V_{c c}^{\prime}=A G_{T}\left(1-B \cdot e^{C V_{c c}}\right)
$$

$$
\begin{aligned}
& A=\frac{\# \text { series } \cdot C_{1} \cdot n_{p} \cdot N}{C \cdot \# \text { parallel }} \\
& B=e^{\frac{-e\left(V_{o c}\right)}{m k T}} \\
& C=\frac{e}{m k T}
\end{aligned}
$$

And the solution is:

$$
\begin{gathered}
-\frac{1}{C} \ln \left[\frac{-B C e^{C V_{c c}}+C}{e^{C V_{c c}}}\right]=A \int G_{T} d t= \\
a_{0}^{0} a_{0}^{1} x+a_{0}^{0} a_{2}^{1} \frac{L_{1}}{2 \pi} \operatorname{sen} \frac{2 \pi x}{L_{1}}+a_{0}^{0} a_{4}^{1} \frac{L_{1}}{4 \pi} \operatorname{sen} \frac{4 \pi x}{L_{1}}+a_{0}^{0} a_{6}^{1} \frac{L_{1}}{6 \pi} \operatorname{sen} \frac{6 \pi x}{L_{1}}+ \\
a_{2}^{0} a_{2}^{1} \frac{L_{0}}{2 \pi} \operatorname{sen} \frac{2 \pi x}{L_{0}}+a_{2}^{0} a_{2}^{1} \frac{L_{0}}{2 \pi}\left(\frac{\operatorname{sen}\left(\frac{2 \pi}{L_{0}}-\frac{2 \pi}{L_{1}}\right) x}{2\left(\frac{2 \pi}{L_{0}}-\frac{2 \pi}{L_{1}}\right)}+\frac{\operatorname{sen}\left(\frac{2 \pi}{L_{0}}+\frac{2 \pi}{L_{1}}\right) x}{2\left(\frac{2 \pi}{L_{0}}+\frac{2 \pi}{L_{1}}\right)}\right)+\ldots .
\end{gathered}
$$

Equation 8: solving DC voltage in charging mode At this moment we have obtained an expression for the DC voltage in the ultracapacitors bank. Based on it it's possible to obtain the current that flow by them, instant power ant the energy stored in every time.

State 2: Ultracapacitors in discharging mode $I_{i} \neq 0$

$$
I_{c}=I_{p}-I_{i}
$$

so:

$$
V_{c c}=V_{c c \max }+\frac{\# \text { series }}{C \cdot \# \text { parallel }} \int I_{p}-\frac{P}{V_{c c}} d t
$$

where $\mathrm{P}=$ Inverter output power

Equation 9: DC voltage in discharging mode In the same way that state 1 , we can express

$$
D=\frac{n P}{C}
$$

and we will obtain the next differential equation:

$$
V_{c c}^{\prime}=A G_{T}\left(1-B \cdot e^{C V_{c c}}\right)-\frac{D}{V_{c c}}
$$

Equation 10: DC voltage in charging mode This equation has no analitic solution and we only can obtain a numeric solution. However we can do a simplification that can be good enough for few cycles, considering small variations of the DC voltage, near to the MPP: that means the consideration of constant voltage. The value taken is the media value of DC voltage range. In this way the equation can be solved integring directly:

$V_{c c}=\int\left(A G_{T}\left(1-B \cdot e^{C V_{c c}}\right)-\frac{D}{V_{c c}}\right) d t=\left(1-B \cdot e^{C V_{c c}}\right) A \int G_{T}-\frac{D t}{V_{c c}}$

Equation 11:aprox. of DC voltage. Charging mode This equation 11 is the base to calculate an aproximation of the current that flow in the ultracapacitors batery discharge, power and energy stored every time.

\section{Sizing the system}

There are three aspects to consider:

a) The energy stored by the ultracapacitors is:

The balance of the system is:

$$
E p=E c+E i
$$

where:

$\mathrm{Ep}=\mathrm{PV}$ input energy and

$\mathrm{Ei}=$ energy extracted by the inverter, 
If Ep $>$ Ei the the system will react, lowing the PV power:

- Rising the DC voltage or

- Cutting off the PV modules. This situation will give a lower performance.

In order to well know this situation is necessary to analize the irradiance of the placement. This situation can be in the days with the highest annual irradiance. Experimentally we can assure that a good criteria to choose the output inverter power is the next:

$P_{i}=\frac{\# P V \bmod \text { ules } \times \text { Surface } \times \text { Maximum_daily_Energy } \times P V_{-} \text {performance }}{\# \text { of_daily_hours }}$

b) The discharging time has a short relation when we consider the dead times when the system exceed the supplied energy. It's easy to calculate with next equation. Also it can provides us the minimum and the maximum time of the inverter working at constant output power.

$$
T_{\text {des }}=\frac{E_{c}}{P_{i}}=\frac{\frac{1}{2} n C(\Delta V)^{2}}{P_{i}} \leq T_{\min }
$$

c) The performance of the inverter. We can use a good approximation if we choose:

$$
\approx 30 \% P_{n} \leq P_{i} \leq P_{n}
$$

An evaluation of these three aspects will provide us a good idea of the output power.

\section{Strategies of control}

We present four strategies to control the system. One of them is a conventional system without ultracapacitors in order to compare the results with another three strategies using those elements.

a) System 1: Conventional system: It works at variable power. Starting at a minimum input power, and including a MPP tracking

b) System 2: Using ultracapacitors at variable voltage depending of the ultracapacitors characteristics. Inverter works at constant ouptput power at its maximum eficiency.

c) System 3: Using ultracapacitors at variable voltage. Tracking of the MPP with a DC regulator before the ultracapacitors. Still maximum and minimum working voltages. Inverter works at constant output power at its maximum efficiency.

d) System 4: Same at above but inverter works at two different levels: normal level and upper level for extreme situation (very high input PV power)

e) System 5: Hybrid strategy. We fix a working power. When PV system provides more power, inverter works like a conventional system. When input power is lower, we charge and discharge the ultracapacitors at constant power.

\section{Simulations}

We have done simulations of every strategy control in Matlab at different conditions. We'll provide several graphics of the results. In this abstract we show the

\begin{tabular}{|c|c|c|c|c|c|c|}
\hline & A & $B$ & c & $D$ & $E$ & $\mathrm{~F}$ \\
\hline 1 & $|P| \approx 1000 \mathrm{~W}$ & & & & & \\
\hline 2 & $\cos \varphi=1$ & SISTEMA 1 & SISTEMA 2 & SISTEMA 3 & SISTEMA 4 & SISTEMA 5 \\
\hline 3 & POT & VARIABLE & 700 & 700 & $500-1000$ & 500 - VARIABLE \\
\hline 4 & $p$ & CURVA & 0,912 & 0,912 & 0,91 & 0,92 - CURVA \\
\hline 5 & ENERGIA & 1,1408 & 1,147 & 1,1809 & 1,1965 & 1,2061 \\
\hline 6 & $\%$ & & $0,54 \%$ & $3,52 \%$ & $4,88 \%$ & $5,72 \%$ \\
\hline \multicolumn{7}{|c|}{7} \\
\hline 8 & $\cos \varphi=0,7$ & SISTEMA 1 & SISTEMA 2 & SISTEMA 3 & SISTEMA 4 & SISTEMA 5 \\
\hline 9 & POT & VARIABLE & 700 & 700 & $500-1000$ & 500 - VARIABLE \\
\hline 10 & $\rho$ & CURVA & 0,878 & 0,878 & 0,8725 & 0,89 - CURVA \\
\hline 11 & ENERGÍA & 1,1072 & 1,1043 & 1,1369 & 1,148 & 1,1651 \\
\hline 12 & $\%$ & & $-0,26 \%$ & $2,68 \%$ & $3,68 \%$ & $5,23 \%$ \\
\hline \multicolumn{7}{|c|}{13} \\
\hline 14 & $\cos \varphi=0,4$ & SISTEMA 1 & SISTEMA 2 & SISTEMA 3 & SISTEMA 4 & SISTEMA 5 \\
\hline 15 & POT & VARIABLE & 700 & 700 & $500-1000$ & 500 - VARIABLE \\
\hline 16 & $p$ & CURVA & 0,759 & 0,759 & 0,765 & 0,795 - CURVA \\
\hline 17 & ENERGIA & 0,99826 & 0,9546 & 0,98281 & 1,0058 & 1,0309 \\
\hline 18 & $\%$ & & $-4,37 \%$ & $-1,55 \%$ & $0,76 \%$ & $3,27 \%$ \\
\hline \multicolumn{7}{|c|}{19} \\
\hline \multicolumn{7}{|c|}{20} \\
\hline \multicolumn{7}{|c|}{$21 \quad \mathrm{Pl} \approx 2000 \mathrm{~W}$} \\
\hline 22 & $\cos \varphi=1$ & SISTEMA 1 & SISTEMA 2 & SISTEMA 3 & SISTEMA 4 & SISTEMA 5 \\
\hline 23 & POT & VARIABLE & 700 & 700 & $500-1000$ & 500 - VARIABLE \\
\hline 24 & $p$ & CURVA & 0,9258 & 0,9258 & 0,9251 & 0,9277 - CURVA \\
\hline 25 & ENERGIÁA & 1,1106 & 1,1644 & 1,1988 & 1,2163 & 1,2185 \\
\hline 26 & $\%$ & & $4,84 \%$ & $7,94 \%$ & $9,52 \%$ & $9,72 \%$ \\
\hline \multicolumn{7}{|c|}{27} \\
\hline 28 & $\cos \varphi=0,7$ & SISTEMA 1 & SISTEMA 2 & SISTEMA 3 & SISTEMA 4 & SISTEMA 5 \\
\hline 29 & POT & VARIABLE & 700 & 700 & $500-1000$ & 500 - VARIABLE \\
\hline 30 & $p$ & CURVA & 0,892 & 0,892 & 0,892 & \begin{tabular}{|c}
0,899 - CURVA \\
\end{tabular} \\
\hline 31 & ENERGIA & 1,0862 & 1,1219 & 1,15503 & 1,1728 & 1,1788 \\
\hline 32 & $\%$ & & $3,29 \%$ & $6,34 \%$ & $7,97 \%$ & $8,53 \%$ \\
\hline \multicolumn{7}{|c|}{33} \\
\hline 34 & $\cos \varphi=0,4$ & SISTEMA 1 & SISTEMA 2 & SISTEMA 3 & SISTEMA 4 & SISTEMA 5 \\
\hline 35 & POT & VARIABLE & 700 & 700 & $500-1000$ & 500 - VARIABLE \\
\hline 36 & $p$ & CURVA & 0,822 & 0,822 & 0,825 & $\begin{array}{ll}5 & 0,84 \text { - CURVA }\end{array}$ \\
\hline 37 & ENERGIA & 1,025 & 1,0338 & 1,0644 & 1,0847 & 1,0971 \\
\hline 38 & $\%$ & & $0,86 \%$ & $3,84 \%$ & $5,82 \%$ & $7,03 \%$ \\
\hline
\end{tabular}
results in a table

\section{Conclusion}

System 5 provides the best efificiency rate in every condition, so ultracapcitors can be a good solution to increase the production of a Photvoltaic sytem.

\section{References}

[1] Bobby Maher, "A Backup Power System Using Ultracapacitors", september 2004, pags 44-49 www.powerelectronics.com.

[2] NESS Capacitor Co. Ltd, several technical datas

[3] MAXWELL Technologies ultracapacitors, several technical datas

[4] L. Zubieta, R. Bonert, "Characterization of doublelayer capacitors for power electronics applications", IEEE Transactions on Industry Applications, JanuaryFebruary 2000, vol. 36, issue. 1, pp. 199-205. 